Copyright by the American Institute of Physics (AIP). Rao, Wei-Feng; Wang, Yu U., "Bridging domain mechanism for phase coexistence in morphotropic phase boundary ferroelectrics," Appl. Phys. Lett. 90, 182906 (2007); http:// dx.doi.org/10.1063/1.2736276

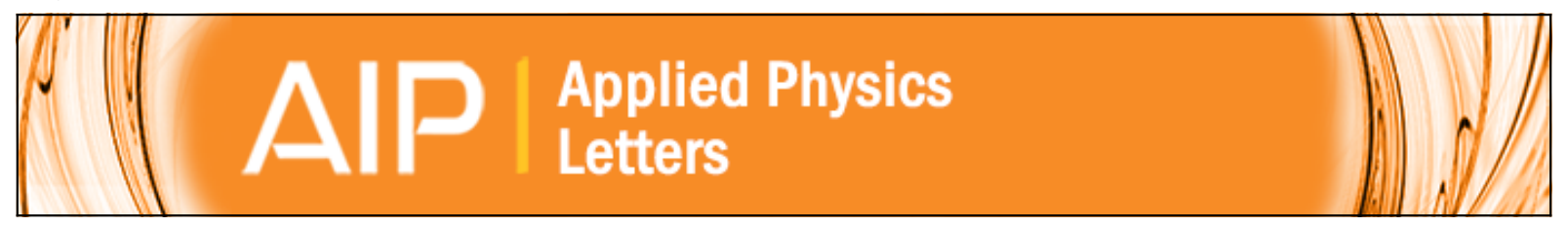

Bridging domain mechanism for phase coexistence in morphotropic phase boundary ferroelectrics

Wei-Feng Rao and Yu U. Wang

Citation: Applied Physics Letters 90, 182906 (2007); doi: 10.1063/1.2736276

View online: $\mathrm{http}: / / \mathrm{dx}$.doi.org/10.1063/1.2736276

View Table of Contents: http://scitation.aip.org/content/aip/journal/apl/90/18?ver=pdfcov

Published by the AIP Publishing

Over 700 papers \&

presentations on

multiphysics simulation vew now

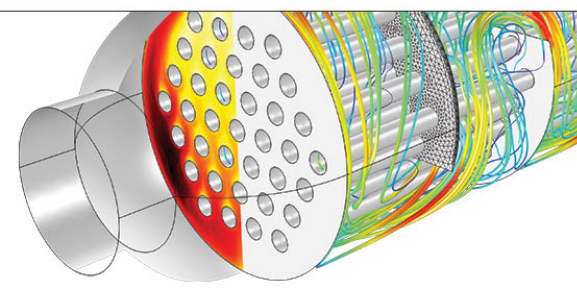




\title{
Bridging domain mechanism for phase coexistence in morphotropic phase boundary ferroelectrics
}

\author{
Wei-Feng Rao and Yu U. Wang ${ }^{\text {a) }}$ \\ Department of Materials Science and Engineering, Virginia Tech, Blacksburg, Virginia 24061
}

(Received 2 April 2007; accepted 8 April 2007; published online 3 May 2007)

\begin{abstract}
Computer modeling and simulation reveals a bridging domain mechanism that explains the phase coexistence commonly observed around the morphotropic phase boundaries of ferroelectric solid solutions. The simulation takes into account the important role of coupled ferroelectric and ferroelastic domain microstructures in phase coexistence phenomenon. It shows that minor domains of metastable phase spontaneously coexist with and bridge major domains of stable phase to reduce total system free energy, including bulk free energy, domain wall energy, and long-range electrostatic and elastostatic energies. The existence of bridging domains also explains the enhanced piezoelectric response in the vicinity of morphotropic phase boundaries. (C) 2007 American Institute of Physics. [DOI: 10.1063/1.2736276]
\end{abstract}

Phase coexistence is commonly observed around the morphotropic phase boundaries (MPBs) of ferroelectric solid solutions. In the temperature-composition phase diagrams of lead-based perovskite-type systems, such as $\mathrm{Pb}\left(\mathrm{Zr}_{1-x} \mathrm{Ti}_{x}\right) \mathrm{O}_{3} \quad(\mathrm{PZT}),{ }^{1} \quad \mathrm{~Pb}\left[\left(\mathrm{Zn}_{1 / 3} \mathrm{Nb}_{2 / 3}\right)_{1-x} \mathrm{Ti}_{x}\right] \mathrm{O}_{3},{ }^{2}$ and $\mathrm{Pb}\left[\left(\mathrm{Mg}_{1 / 3} \mathrm{Nb}_{2 / 3}\right)_{1-x} \mathrm{Ti}_{x}\right] \mathrm{O}_{3},{ }^{3}$ the MPB is a nearly vertical phase boundary separating two ferroelectric phases, namely, rhombohedral and tetragonal phases at low and high Ti contents, respectively. The presence of MPB as a sharp-line phase boundary has raised great scientific curiosity, because it violates Gibbs phase rule for thermodynamically equilibrium temperature-composition phase diagram, ${ }^{4}$ and the mechanism of phase transition between ferroelectric rhombohedral and tetragonal phases across MPB is an interesting question. ${ }^{5}$ Because the best piezoelectric properties of these materials are obtained at compositions in the vicinity of their MPBs, ${ }^{1,6-8}$ the effect of MPB phase coexistence on the piezoelectric properties is a question of both scientific and technological importance. Recently, new intermediate MPB phases were observed by high-resolution x-ray and neutron diffractions, ${ }^{9}$ which renews the interest in the phase diagrams of these ferroelectric solid solutions.

As the current material of choice for a wide variety of high-performance electromechanical devices, PZT has been best studied. Despite the investigation of over four decades, the origin of MPB phase coexistence and its effect on the increased piezoelectric response near MPB composition still remain as an open question. Insights have been gained from different perspectives, e.g., stability and metastability of coexisting phases, ${ }^{10}$ solubility gap, ${ }^{11}$ compositional fluctuation, ${ }^{12}$ statistical distribution of accessible polar states, ${ }^{13}$ progression of local structural order, ${ }^{5}$ and equilibrium phase diagram satisfying Gibbs phase rule. ${ }^{4}$ While these studies together provide a broad aspect of MPB phase coexistence phenomenon, none of them considers the formation of ferroelectric and ferroelastic domain microstructures and its role in phase coexistence. That is, the domain microstructure-dependent long-range electrostatic and elastostatic interactions are not considered. The purpose of this

a) Author to whom correspondence should be addressed; electronic mail: yuwang@mse.vt.edu letter is to report a bridging domain mechanism for formation of mosaic domain microstructures that explains the phase coexistence phenomenon and enhanced piezoelectricity around MPBs.

Spontaneous domain formation is an essential feature of ferroelectrics, where the spontaneous polarization selfarranges into head-to-tail patterns to minimize the long-range electrostatic interaction energy, and the accompanying ferroelastic lattice distortion is self-accommodated through formation of structural twins to minimize the long-range elastostatic interaction energy. While twin formation well accommodates the lattice misfit among multiple structural orientation variants of single phase (rhombohedral or tetragonal), adjoining rhombohedral and tetragonal domains across a coherent interface does not accommodate the lattice misfit between them, thus a stress-free state cannot be achieved. Also, the polarization distribution in the interphase interface region needs to be investigated. Clearly, the electrostatic and elastostatic interactions have significant effects on the formation of coupled ferroelectric and ferroelastic domain microstructures and on the phase coexistence around MPBs. In this work we employ computer modeling and simulation to study the interplays among various energetic contributions, including electrostatic energy of polarization distribution, elastostatic energy of lattice misfit, gradient energy of polarization change across domain walls, and bulk energy gap between metastable and stable phases. In particular, we investigate the domain microstructures and mechanisms for phase coexistence and piezoelectricity enhancement around MPBs.

Phase-coexisting ferroelectrics are spatially heterogeneous systems with multiphase and multidomain microstructures. The heterogeneity can originate from two physically distinct processes, i.e., diffusional and diffusionless. Diffusional process leads to compositional fluctuation ${ }^{12}$ or decomposition into two-phase equilibrium. ${ }^{4}$ Since diffusion kinetics in the complex oxide solid solutions is slow at room temperature, diffusional process is expected to play an important role only at elevated temperature or over long time period. In this work we focus on the diffusionless mechanism, i.e., the displacive evolution of spontaneous polarization leading to the formation of domain microstructure without local composition change. 
The multidomain state of a phase-coexisting, compositionally homogeneous ferroelectric system is described by the polarization field $\mathbf{P}(\mathbf{r})$, whose total system free energy is $^{14-16}$

$$
\begin{aligned}
F= & \int d^{3} r\left[f(\mathbf{P})+\frac{1}{2} \beta_{i j k l} \nabla_{i} P_{j} \nabla_{k} P_{l}\right] \\
& +\frac{1}{2} \int \frac{d^{3} k}{(2 \pi)^{3}}\left[\frac{n_{i} n_{j}}{\varepsilon_{0}} \widetilde{P}_{i} \widetilde{P}_{j}^{*}+K_{i j k l} \widetilde{\varepsilon}_{i j}^{0} \widetilde{\varepsilon}_{k l}^{*}\right],
\end{aligned}
$$

where summation convention over repeated indices is implied. The function $f(\mathbf{P})$ is the nonequilibrium local bulk free energy density that defines the stress-free single-domain thermodynamic properties of all possible coexisting (stable and metastable) ferroelectric phases, and is formulated by Landau-Ginzburg-Devonshire (LGD) theory: ${ }^{17}$

$$
\begin{aligned}
f(\mathbf{P})= & \alpha_{1}\left(P_{1}^{2}+P_{2}^{2}+P_{3}^{2}\right)+\alpha_{11}\left(P_{1}^{4}+P_{2}^{4}+P_{3}^{4}\right)+\alpha_{12}\left(P_{1}^{2} P_{2}^{2}\right. \\
& \left.+P_{2}^{2} P_{3}^{2}+P_{3}^{2} P_{1}^{2}\right)+\alpha_{111}\left(P_{1}^{6}+P_{2}^{6}+P_{3}^{6}\right) \\
& +\alpha_{112}\left[P_{1}^{4}\left(P_{2}^{2}+P_{3}^{2}\right)+P_{2}^{4}\left(P_{3}^{2}+P_{1}^{2}\right)+P_{3}^{4}\left(P_{1}^{2}+P_{2}^{2}\right)\right] \\
& +\alpha_{123} P_{1}^{2} P_{2}^{2} P_{3}^{2},
\end{aligned}
$$

where the expansion coefficients $\alpha_{i}, \alpha_{i j}$, and $\alpha_{i j k}$ are determined by fitting the theoretical predictions to experimental data. For PZT system, the experimentally determined coefficients are functions of composition $x$ (i.e., mole fraction of $\mathrm{PbTiO}_{3}$ ) and temperature $T .{ }^{18}$ The gradient term in Eq. (1) characterizes the energy contribution associated with polarization change across domain walls and interphase interfaces, where the tensor $\beta_{i j k l}$ is the gradient coefficient and $\nabla_{i}=\partial / \partial r_{i}$ is gradient operator. The $\mathbf{k}$-space integral in Eq. (1) gives the electrostatic energy of polarization distribution $\mathbf{P}(\mathbf{r})$ and the elastostatic energy of misfit strain distribution $\boldsymbol{\varepsilon}^{0}(\mathbf{r})$, where $\varepsilon_{0}$ is the permittivity of free space, $K_{i j k l}=C_{i j k l}$ $-n_{m} C_{i j m n} \Omega_{n p} C_{k l p q} n_{q}, \Omega_{i k}=\left(C_{i j k l} n_{j} n_{l}\right)^{-1}, C_{i j k l}$ is the elastic modulus tensor, and $\mathbf{n}=\mathbf{k} / k$. The spontaneous lattice misfit strain is coupled to polarization through electrostriction coefficient tensor $Q_{i j k l}, \varepsilon_{i j}^{0}=Q_{i j k l} P_{k} P_{l}$. The functions $\widetilde{\mathbf{P}}(\mathbf{k})$ and $\widetilde{\boldsymbol{\varepsilon}}^{0}(\mathbf{k})$ are the Fourier transform of the fields $\mathbf{P}(\mathbf{r})$ and $\boldsymbol{\varepsilon}^{0}(\mathbf{r})$, respectively, and the superscript asterisk ${ }^{*}$ indicates the complex conjugate. The evolution of polarization and domain microstructure is characterized by the time-dependent Ginzburg-Landau equation ${ }^{14-16}$

$$
\frac{\partial P_{i}(\mathbf{r}, t)}{\partial t}=-L_{i j} \frac{\delta F}{\delta P_{j}(\mathbf{r}, t)}+\xi_{i}(\mathbf{r}, t),
$$

where $L_{i j}$ is the kinetic coefficient tensor, and $\xi_{i}(\mathbf{r}, t)$ is Gaussian-distributed Langevin noise term to account for the effect of thermal fluctuation. In this work, we use $\beta_{i j k l}$ $=\beta \delta_{i k} \delta_{j l}, L_{i j}=L \delta_{i j}$, and the experimentally determined LGD polynomial coefficients, ${ }^{18}$ ultrasonically measured elastic constants, ${ }^{19}$ and Rietveld refinement-derived electrostriction coefficients $^{20}$ for PZT system at room temperature within MPB composition range.

The computer simulation reveals a bridging domain mechanism for phase coexistence around MPB, as shown in Fig. 1. We consider PZT system at room temperature with near MPB compositions. Figure 1(a) shows the simulated domain microstructure in PZT of composition $x=0.49$. The simulation shows that minor domains of tetragonal phase (green) spontaneously coexist with and bridge major do-

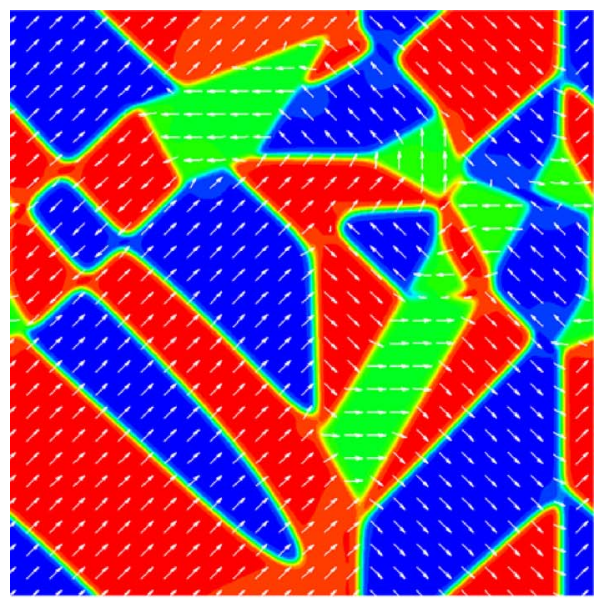

(a)

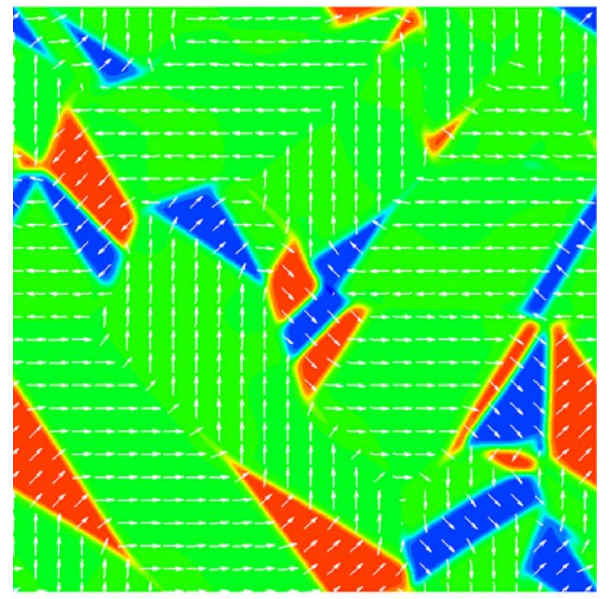

(b)

FIG. 1. (Color online) Computer simulation of bridging domains and mosaic domain microstructures of coexisting phases in PZT system at room temperature with near MPB compositions: (a) $x=0.49$ and (b) $x=0.50$. Arrows represent the in-plane components of polarization vector, and the outof-plane component is represented by color, where green domains are tetragonal phase and red and blue domains are rhombohedral phase. Minor domains of metastable phase spontaneously coexist with and bridge major domains of stable phase and together form mosaic domain microstructures to reduce total system free energy. The coupled ferroelectric and ferroelastic domain microstructures lead to spontaneous phase coexistence in compositionally homogeneous system around MPB.

mains of rhombohedral phase (red and blue), and together form mosaic domain microstructure. Figure 1(b) shows the simulated domain microstructure in PZT of composition $x=0.50$. In contrast to the case of $x=0.49$ shown in Fig. 1(a), the rhombohedral phase (red and blue) forms minor domains that coexist with and bridge the major domains of tetragonal phase (green). Such phase-coexisting mosaic domain microstructures effectively reduce the total system free energy, including bulk free energy, domain wall energy, and longrange electrostatic and elastostatic energies.

In the simulated phase-coexisting mosaic domain microstructures (Fig. 1), the domains of tetragonal phase form structural (ferroelastic) twins of $\{110\}$ twin planes, where the twin boundaries are also $90^{\circ}$ ferroelectric domain walls; the domains of rhombohedral phase form twins of either $\{100\}$ or $\{110\}$ twin planes, where the twin boundaries are $109^{\circ}$ and $71^{\circ}$ ferroelectric domain walls, respectively. This observation is in agreement with the crystallographic analysis of domain microstructures simultaneously minimizing elastostatic and 
electrostatic energies. The more interesting observation, however, is the spontaneous coexistence of minor phase that bridges the domains of major phase. The existence of minor phase as bridging domains reduces both elastostatic energy, electrostatic energy, and polarization gradient energy in complex domain microstructures, which arise from the frustrations caused by the crystal lattice misfit among multiple structural orientation variants and the polarization distribution among multiple polar axes. It is worth noting that the transformation twinning mechanism can completely selfaccommodate the lattice misfit only within one set of twinrelated lamellar domains composed of two structural variants with same twin plane orientation, i.e., polytwin. ${ }^{21} \mathrm{~A}$ coherent domain microstructure consisting of multiple structural variants inevitably generates disclinationlike and/or dislocationlike internal stresses, ${ }^{21}$ which stabilize the bridging domains of minor phase, leading to phase coexistence. In particular, bridging domain forms between the domains of major phase that do not form structural twin or head-to-tail polarization pattern. Bridging domain also provides a low-energy pathway for polarization transition between different polar axes of the major phase. As shown in Fig. 1, the interphase interfaces exhibit preferred orientations, which are close to either $\{350\}$ or $\{250\}$ in two-dimensional simulation. Analysis shows that such interface orientations minimize the interfacial charge density, i.e., $\left(\mathbf{P}^{R}-\mathbf{P}^{T}\right) \cdot \mathbf{n} \approx 0$, where $\mathbf{n}$ is the interface normal vector, and $\mathbf{P}^{R}$ and $\mathbf{P}^{T}$ are the polarization vectors in the neighboring rhombohedral and tetragonal domains, respectively. In particular, $\{350\}$ and $\{250\}$ interphase interfaces are respectively $55^{\circ}$ and $125^{\circ}$ domain walls between rhombohedral and tetragonal phases. As shown in Fig. 1, the characteristic triangular bridging domains have two $\{350\}$ and one $\{250\}$ interfaces. Three-dimensional simulation is under way to investigate the polyhedral shapes and interface orientations of the bridging domains.

Formation of bridging domains of minor phase increases the bulk free energy of the ferroelectric system. In the vicinity of MPB, the bulk energy gap between rhombohedral and tetragonal phases is small, thus the bulk free energy increase is overcounterbalanced by the decreases of elastostatic, electrostatic, and polarization gradient energies, leading to reduction in total system free energy. With compositions increasingly deviated from the MPB, the bulk energy gap becomes increasingly large, resulting in prohibitively high energy cost for minor phase formation. Our simulations with compositions $x=0.48$ and $x=0.51$ show diminishing amount of minor phase and bridging domains. Therefore, bridging domain mechanism can operate only in the vicinity of MPB and lead to phase coexistence around MPB. Phase coexistence due to bridging domain mechanism is expected for a wider composition range around MPB in polycrystalline ceramics than in single crystals, because grain boundary constraints generate larger internal stresses in polycrystal grains, as has been investigated in ferroelastic (martensitic) systems. ${ }^{22,23}$ The larger internal stresses tend to stabilize the bridging domains of minor phases over a wider composition range around MPB. Phase coexistence in ferroelectric ceramics is under investigation as ongoing research by using computer modeling of polycrystals similar to the models previously developed for martensites and ferromagnetics. ${ }^{22-24}$
The spontaneously formed phase-coexisting mosaic domain microstructure corresponds to an energy-minimizing state. An externally applied electric field can induce a configurational change in the domain microstructure, deviating from the local minimum-energy state. The field-induced microstructure will automatically return to its original minimum-energy state upon removal of external field. Such a ferroelectric shape memory effect produces large macroscopic electromechanical response, which explains the enhanced piezoelectricity around MPB. Detailed simulation studies of ferroelectric shape memory effect will be reported in a separate publication.

In summary, computer modeling and simulation at compositions near MPB reveals a bridging domain mechanism, whereby major and minor phases spontaneously coexist and form mosaic domain microstructures without compositional inhomogeneity. A ferroelectric shape memory effect resulting from the spontaneously formed minimum-energy multidomain state explains the enhanced piezoelectric responses around MPB.

The parallel computer simulations were performed on Data Star at San Diego Supercomputer Center and System X at Virginia Tech.

${ }^{1}$ B. Jaffe, W. R. Cook, and H. Jaffe, Piezoelectric Ceramics (Academic, London, 1971).

${ }^{2}$ J. Kuwata, K. Uchino, and S. Nomura, Ferroelectrics 37, 579 (1981).

${ }^{3}$ S. W. Choi, T. R. Shrout, S. J. Jang, and A. S. Bhalla, Ferroelectrics 100, 29 (1989).

${ }^{4}$ G. A. Rossetti, Jr., W. Zhang, and A. G. Khachaturyan, Appl. Phys. Lett. 88, 072912 (2006).

${ }^{5}$ A. M. Glazer, P. A. Thomas, K. Z. Baba-Kishi, G. K. H. Pang, and C. W. Tai, Phys. Rev. B 70, 184123 (2004).

${ }^{6}$ J. Kuwata, K. Uchino, and S. Nomura, Jpn. J. Appl. Phys. 21, 1298 (1982).

${ }^{7}$ T. R. Shrout, Z. P. Chang, N. Kim, and S. Markgraf, Ferroelectr., Lett. Sect. 12, 63 (1990).

${ }^{8}$ S. E. Park and T. R. Shrout, J. Appl. Phys. 82, 1804 (1997).

${ }^{9}$ For complete references to the new intermediate MPB phases, see recent review in B. Noheda and D. E. Cox, Phase Transitions 79, 5 (2006).

${ }^{10}$ V. A. Isupov, Sov. Phys. Solid State 10, 989 (1968) [Fiz. Tverd. Tela (Leningrad) 10, 1244 (1968)]; Phys. Solid State 43, 2262 (2001) [Fiz. Tverd. Tela (S.-Peterburg) 43, 2171 (2001)]; Ferroelectrics 266, 91 (2002).

${ }^{11}$ L. Benguigui, Solid State Commun. 11, 825 (1972); P. Ari-Gur and L. Benguigui, ibid. 15, 1077 (1974).

${ }^{12}$ K. Kakegawa, J. Mohri, T. Takahashi, H. Yamamura, and S. Shirasaki, Solid State Commun. 24, 769 (1977); K. Kakegawa, J. Mohri, S. Shirasaki, and K. Takahashi, J. Am. Ceram. Soc. 65, 515 (1982).

${ }^{13}$ W. Cao and L. E. Cross, Phys. Rev. B 47, 4825 (1993).

${ }^{14}$ S. Semenovskaya and A. G. Khachaturyan, J. Appl. Phys. 83, 5125 (1998).

${ }^{15}$ Y. L. Li, S. Y. Hu, Z. K. Liu, and L. Q. Chen, Appl. Phys. Lett. 78, 3878 (2001).

${ }^{16}$ W. F. Rao and Y. U. Wang, Appl. Phys. Lett. 90, 041915 (2007).

${ }^{17}$ A. F. Devonshire, Philos. Mag. 40, 1040 (1949).

${ }^{18}$ A. Amin, M. J. Haun, B. Badger, H. McKinstry, and L. E. Cross, Ferroelectrics 65, 107 (1985).

${ }^{19}$ T. Fett and D. Munz, J. Test. Eval. 28, 27 (2000).

${ }^{20}$ W. F. Rao and Y. U. Wang (unpublished).

${ }^{21}$ L. A. Bendersky, A. Roytburd, and W. J. Boettinger, Acta Metall. Mater. 42, 2323 (1994).

${ }^{22}$ Y. M. Jin, A. Artemev, and A. G. Khachaturyan, Acta Mater. 49, 2309 (2001).

${ }^{23}$ Y. U. Wang, Y. M. Jin, and A. G. Khachaturyan, Acta Mater. 52, 1039 (2004).

${ }^{24}$ Y. M. Jin, Y. U. Wang, A. Kazaryan, Y. Wang, D. E. Laughlin, and A. G. Khachaturyan, J. Appl. Phys. 92, 6172 (2002). 